\title{
Sphingosine kinase inhibition exerts both pro- and anti-atherogenic effects in low-density lipoprotein receptor-deficient (LDL- ${ }^{-/-}$) mice
}

\author{
Francesco Potì ; Martine Bot²; Sara Costa'; Valeria Bergonzini'; Lynn Maines ${ }^{3}$; Georg Varga4; Hendrik Freise ${ }^{5}$; Horst Robenek ${ }^{6}$; \\ Manuela Simoni ${ }^{1 *}$; Jerzy-Roch Nofer ${ }^{1,7^{*}}$ \\ ${ }^{1}$ Department of Medicine, Endocrinology, Metabolism and Geriatrics, University of Modena and Reggio Emilia, Modena, Italy; ${ }^{2}$ Division of Biopharmaceutics, Gorlaeus Leiden/ \\ Amsterdam Center for Drug Research, Gorlaeus Laboratories, Leiden University, Leiden, The Netherlands; ${ }^{3}$ Apogee Biotechnology Corporation, Hershey, Pennsylvania, USA; \\ ${ }^{4}$ Institute of Immunology, University of Münster, Münster, Germany; ${ }^{5}$ Department of Anaesthesiology and Intensive Medicine, University Hospital Münster, Münster, Germany; \\ ${ }^{6}$ Leibniz-Institute for Arteriosclerosis Research, University of Münster, Münster, Germany; ${ }^{7}$ Center for Laboratory Medicine, University Hospital Münster, Münster, Germany
}

\begin{abstract}
Summary
Sphingosine 1-phosphate (S1P), a lysosphingolipid associated with high-density lipoprotein (HDL), contributes to the anti-atherogenic potential attributed to this lipoprotein. This study examined whether a reduction of S1P plasma levels affects atherosclerosis in a murine model of disease. LDL- $R^{-1-}$ mice on Western diet were given ABC294640, an inhibitor of sphingosine kinase (SphK) for 16 weeks. $A B C 294640$ decreased plasma S1P by approximately 30\%. However, ABC294640 failed to affect atherosclerotic lesion formation. Plasma triglycerides were reduced whereas total and $\mathrm{HDL}$-cholesterol remained unchanged in course of $A B C 294640$ treatment. $A B C 294640$ increased plasma interleukin (IL)-12p70 and RANTES concentration as well as IL-12p70, RANTES and interferon (IFN)- $\gamma$ production by peritoneal cells and this was paralleled by enhanced activity of peritoneal and spleen dendritic cells as evidenced by up-regulation of CD86 and MHC-II on CD11 $\mathrm{C}^{+}$ cells. As a consequence, increased T-cell activation was noted in ABC294640-treated mice as indicated by enhanced CD4 ${ }^{+}$splenocyte
\end{abstract}

proliferation, IFN- $\gamma$ and IL-2 production, and CD69 expression. Concomitantly, however, $\mathrm{ABC} 294640$ treatment redistributed $\mathrm{CD}^{+}$and $\mathrm{CD}^{+}$cells from blood to lymphatic organs and reduced T-cell number within atherosclerotic lesions. In addition, plasma SVCAM-1, sICAM-1, and MCP-1 levels as well as in vivo leukocyte adhesion and CCL19-induced T-cell penetration into peritoneum were lower in ABC294640-treated animals. In vitro experiments demonstrated reduced VCAM-1 and ICAM-1 expression and lymphocyte adhesion to endothelial cells exposed to $A B C 294640$. In conclusion, treatment with SphK inhibitor leads to both pro- and anti-atherogenic effects in LDL- $\mathrm{R}^{-1-}$ mice. As a consequence, SphK inhibition fails to affect atherosclerosis despite significant S1P reduction in plasma.

\section{Keywords}

Sphingosine 1-phosphate, high density lipoproteins, inflammation, endothelium, animal models of atherosclerosis

\section{Financial support:}

This study was supported by Grant N0110816 from the Innovative Medizinische Forschung (IMF) to J.-R.N, a grant IDEAS RBID08777T from the Italian Ministry of Education, Universities and Research to J.-R.N. and M.S., and intramural resources of the Center for Laboratory Medicine to J.-R.N.

Received: September 4, 2011

Accepted after minor revision: November 29, 2011

Prepublished online: January 11, 2012

doi:10.1160/TH11-08-0583

Thromb Haemost 2012; 107: 552-561

\section{Introduction}

Sphingosine 1-phosphate (S1P) is an important lipid mediator generated from phospholipids upon cell activation and is present in plasma and extracellular fluid in high nanomolar concentrations $(200-1,000 \mathrm{nM})(1,2)$. S1P is produced by phosphorylation of sphingosine by sphingosine kinases (SphK) 1 and 2 in response to a variety of stimuli and is actively exported out of the cells (inside-out signalling), where it serves as a ligand for five $G$ protein-coupled receptors termed S1PR1-5 (3-5). Extracellular S1P exerts modulatory effects on diverse processes in the vasculature including survival, proliferation, migration and cell-cell adhesion of endothelial and smooth muscle cells and thereby con- trols endothelial barrier integrity, vascular development and vessel contraction $(6,7)$. Moreover, S1P regulates lymphocytes and monocytes trafficking between lymphoid compartments and peripheral sites of inflammation and promotes development of antiinflammatory functional cell phenotypes (Th2, M2) (8-10). In addition to extracellular effects S1P assumes a role as an intracellular second messenger and promotes expression of cytokines and adhesion-mediating molecules such as VCAM-1 and ICAM-1 in macrophages and endothelial cells, respectively $(1,11,12)$. Due to these pleiotropic effects exerted both extra- and intracellulary, S1P is critical for the maintenance of vascular homeostasis, while its perturbations may be decisive for the emergence of inflammatory vascular diseases such as atherosclerosis. 
Recent investigations provide circumstantial evidence suggesting that S1P may be directly involved in the pathogenesis of atherosclerosis. For instance, extracellular S1P is mainly contained in high-density lipoproteins (HDL) - a potent plasma-borne antiatherogenic factor, and decreased HDL-bound S1P levels have been observed in patients with coronary artery disease and myocardial infarction $(13,14)$. In addition, S1P was found to emulate in vitro several atheroprotective effects attributed to HDL, including inhibition of endothelial apoptosis and stimulation of endothelial cell movement, inhibition of the expression of adhesion molecules and stimulation of nitric oxide (NO) and prostacyclin generation (15-18). FTY720, a synthetic S1P analogue and a high affinity agonist for S1PR1, 3, 4, and 5 was demonstrated to retard atherosclerotic lesion formation in both low-density lipoprotein (LDL) receptor-deficient (LDL- $\mathrm{R}^{-/}$) and apolipoprotein E-deficient $\left(\right.$ apoE $\left.^{-/-}\right)$mice fed cholesterol-rich Western diet $(19,20)$. However, the contribution of endogenous S1P to the development of atherosclerosis has not been investigated to date. To address this issue, we studied the development of atherosclerosis in LDL- $\mathrm{R}^{-/}$ mice exposed to ABC294640, a potent and specific SphK inhibitor $(21,22)$. Our results demonstrate that the SphK inhibition and the ensuing reduction of plasma S1P levels lead to both pro-atherogenic effects (via enhanced activation of dendritic cells and T-cells) and anti-atherogenic effects (via reduced activation of endothelial cells) that mutually equalise. As a consequence, SphK inhibition does not affect atherosclerotic lesion formation in LDL- $\mathrm{R}^{-/-}$mice.

\section{Material and methods}

\section{Reagents}

ABC294640 [3-(4-chlorophenyl)-adamantane-1-carboxylic acid (pyridin-4-ylmethyl)amide] was provided by Apogee Biotechnology Corp. (Hummelstown, PA, USA). Cell culture reagents including DMEM, RPMI1640, non-essential amino acids and sodium pyruvate were from Lonza (Wuppertal, Germany). Fetal calf serum (FCS) was from PAA Laboratories (Cölbe, Germany). Unless indicated otherwise, all other chemicals were from Sigma (Deisenhofen, Germany), and were of highest purity available.

\section{Animals}

Female LDL-R $\mathrm{R}^{-/-}$mice on a C57BL/6J background were purchased from Jackson Laboratories (Bar Harbor, ME, USA), and housed under pathogen-free conditions. Mice ( 8 weeks, $\sim 20 \mathrm{~g}$, female) were put on an atherogenic Western diet (0.25\% cholesterol, $21 \%$ fat; Altromin, Lage, Germany) for 16 weeks. Animals were separated randomly into a treatment group receiving five days a week in-

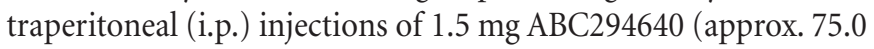
$\mathrm{mg} / \mathrm{kg} /$ day) and a control group injected with the same volume $(0.1 \mathrm{ml})$ of saline. At the end of the treatment period mice were bled by retro-orbital vein puncture under complete anesthesia, peritoneal macrophages were isolated and tissues were collected for further analysis. The experimental protocol was approved by local Committee for Animal Protection.

\section{Intravital microscopy}

For studying leukocyte adhesion under in vivo conditions, $\mathrm{LDL}^{-\mathrm{R}^{-/}}$mice were put on Western diet and administered ABC294640 (75.0 mg/kg/day) or saline for seven days. Leukocyte adhesion was stimulated by i.p. injection of $5.0 \mathrm{mg} / \mathrm{kg}$ endotoxin. After 6 hours (h), leukocytes were stained perivitally with $1.0 \mathrm{mg} /$ $\mathrm{kg}$ of carboxyfluorescein diacetate succinimidyl ester (CFDA-SE, Molecular Probes, Leiden, Netherland) by intravenous injection of the fluorochrome solution as described (23). Animals were anesthetised with $2 \%$ isoflurane, and leukocyte adhesion in mesenterial venoles was assessed by intravital microscopy of small mesenterial venoles (diameter $20-30 \mu \mathrm{m}$ ). For this purpose, a small midline laparotomy was performed in the lower abdomen, animals were placed on their right side on an inverted fluorescence microscope (Eclipse 300, Nikon, Düsseldorf, Germany), and a part of the distal ileum was gently exteriorised. Mesenteric venoles with a diameter of 20-30 $\mu \mathrm{m}$ were visualised and recorded for 60 seconds (sec) by a fluorescence camera (FView II Olympus Soft Imaging Systems, Münster, Germany) on a computer for offline evaluation. Temporary leukocyte adhesion was defined as movement of a leukocyte with markedly reduced speed or adhesion $<20 \mathrm{sec}$ to the endothelium. Permanent adhesion was defined as leukocytes without movement $>20 \mathrm{sec}$. The numbers of leukocytes were normalised on $100 \mu \mathrm{m}$ length of venolar wall.

\section{Histology and lesion analysis}

Exsanguinated animals were subjected to in situ perfusion with saline through the left cardiac ventricle. For analysis of spontaneous atherosclerosis aortic roots were removed and embedded in TissueTek. Transverse $10 \mu \mathrm{m}$ cryosections were prepared, mounted in order on series of slides, and stained with Oil Red $\mathrm{O}$ and hematoxilin (Sigma). Cross-sections with maximal stenosis were used for morphometric analysis on a DM-RE microscope with Leica Qwin image analysis software (Leica Microsystems B.V., Rijswijk, the Netherlands), as described previously (19). Corresponding sections were stained immunohistochemically with antibodies directed against mouse macrophages (monoclonal mouse IgG ${ }_{2 a}$, clone MOMA-2, dilution 1:50; Sigma Diagnostics, St. Louis, MO, USA) and lymphocytes (purified anti-mouse CD3 clone SP7, dilution 1:50; Immunologic, Duiven, The Netherlands). Macrophages positive areas were determined by computer-assisted colour-gated measurement, and related to the total intimal surface area. For lymphocytes, the number of CD3-positive cells was assessed within plaque areas in five consecutive sections, and averages were used for analysis. 


\section{Lipid analysis}

Plasma total cholesterol (TC), HDL-cholesterol (HDL-C) and triglycerides (TG) were determined enzymatically using commercially available kits (Roche, Mannheim, Germany). S1P levels were determined after extraction and derivatisation with $o$-phthaldialdehyde using a Merck-Hitachi LiChrom HPLC system (Merck-Hitachi, Darmstadt, Germany) equipped with RP18 Kromasil column (Chromatographie Service GmbH, Langerwehe, Germany) as previously described (24). The S1P recovery was calculated using dihydro-S1P as a standard.

\section{Leukocyte differential count and lymphocyte subtyping}

Differential leukocyte count was performed manually (Pappenheim staining) in a routine hospital laboratory. Lymphocyte subtyping was performed by flow cytometry. Briefly, whole blood was anti-coagulated with citrate, incubated for 30 minutes ( $\mathrm{min}$ ) with fluorescein isothiocyanate (FITC)- or phycoerythrin (PE)-conjugated antibodies against CD3, CD4, CD8 or CD19 $(5.0 \mu \mathrm{g} / \mathrm{ml}$, Nanotools, Tenningen, Germany), and fixed for 30 min with $0.4 \%$ formaldehyde in phosphate-buffered saline (PBS). Thereafter, cells were centrifuged for $10 \mathrm{~min}$ at 1,700 rpm and erythrocytes were lysed in a buffer containing $0.15 \mathrm{M} \mathrm{NH}_{4} \mathrm{Cl}, 10 \mathrm{mM} \mathrm{NaHCO}_{3}, 0.1$ mM EDTA ( $\mathrm{pH}$ 7.4). The remaining cells were washed twice in PBS and analysed on a FACScalibur flow cytometer (BD Bioscience, San Jose, CA, USA) equipped with a $488 \mathrm{~nm}$ argon laser.

\section{Tissue harvesting}

For chemotaxis studies mice received i.p. injections of CCL19 (500 ng/ml; Peprotech, Rocky Hill, NJ, USA) 16 h before sacrifice. Control mice were injected with PBS. At sacrifice mice were anaesthetised and bled by orbital exsanguination. Peritoneal leukocytes were isolated by peritoneal lavage (ice-cold PBS) as described previously (19). Cells were suspended in DMEM containing FCS $(10.0 \% \mathrm{v} / \mathrm{v})$ and $2 \mathrm{mM}$ glutamine and were either used for flow cytometry or seeded in a 24 -well plate at a density of $0.5 \times 10^{6}$ cells/ $\mathrm{ml}$. After $4 \mathrm{~h}$ non-adherent cells were removed and remaining macrophages were incubated for $24 \mathrm{~h}$ in the absence or presence of lipopolysaccharide (LPS; $50 \mathrm{ng} / \mathrm{ml}$; Sigma). Spleens and lymph nodes (skin and mesenteric) were excised and single-cell suspensions were prepared by passing crude cell suspensions through a 70 $\mu \mathrm{m}$ mesh filter. Erythrocytes in cell suspensions were lysed by hypo-osmotic shock as described above.

\section{Differential cell immunophenotyping}

Peritoneal leukocytes and single-cell suspensions of spleen and lymph nodes were immunophenotyped by flow cytometry (FACSCalibur, BD Biocience, San Jose, CA, USA). Monoclonal antibodies for flow cytometry were from BD Bioscience, Heidelberg, Germany (CD4, CD8 and CD69) or eBioscience, Frankfurt, Germany (CD8, CD19, CD62L, CD11b, CD11c, F4/80, MHCII and CD86). For each FACS staining $2 \times 10^{5}$ cells were incubated with antibody dilutions $(0.25 \mu \mathrm{g}$ for each antibody) in PBS plus $1.0 \%$ (v/v) FCS at $4^{\circ} \mathrm{C}$.

\section{Cytokine determination}

Cytokine (tumour necrosis factor [TNF] $\alpha$, interleukin [IL]12p70, interferon $[\mathrm{IFN}] \gamma, \mathrm{IL}-2)$ and chemokine (MCP-1) levels were quantified in plasma and supernatants of peritoneal leukocytes and splenocytes by commercially available ELISA (R\&D Systems, Wiesbaden, Germany). Semi-quantitative determination of proinflammatory cytokines was performed using a Cytokine Array I from Raybiotech Inc. (Norcross, GA, USA).

\section{T-cell proliferation}

Assessment of T-cell proliferation by flow cytometry was done as described previously (25). Briefly, 1 x $10^{7}$ splenocytes were incubated for $5 \mathrm{~min}$ at $37^{\circ} \mathrm{C}$ in PBS containing $0.5 \mu \mathrm{M}$ carboxyfluorescein diacetate succinimidyl ester (CFSE, Molecular Probes, Leiden, the Netherlands) in the dark, after which cells were washed with PBS containing 1.0\% (v/v) FCS. CFSE-labelled splenocytes were plated, in triplicate, in 96-well plates $\left(1 \times 10^{5}\right.$ cells/well $)$ and either kept in RPMI1640 containing FCS (5.0\% v/v) or stimulated with ConA $(10.0 \mu \mathrm{g} / \mathrm{ml}$, Sigma $)$ or PHA $(5.0 \mu \mathrm{g} / \mathrm{ml}$, Sigma $)$ for five days. Cells were harvested, stained for CD4 and subsequently analysed for proliferation by flow cytometry. Intensity peaks with reduced CFSE fluorescence were used as a measure of splenocyte proliferation.

\section{Endothelial cell culture and in vitro adhesion assay}

Murine endothelial cell line bEnd.5 was a generous gift of Dr. Sigrid März (Max-Planck-Institute for Molecular Medicine, Münster, Germany) and was maintained in DMEM supplemented with glutamine $(2.0 \%, \mathrm{v} / \mathrm{v})$, sodium pyruvate $(1.0 \%, \mathrm{v} / \mathrm{v})$, non-essential amino acids $(1.0 \%, \mathrm{v} / \mathrm{v}), \mathrm{FCS}(20.0 \%, \mathrm{v} / \mathrm{v})$, and endothelial cell growth supplement (PromoCell, Heidelberg, Germany) containing human basic fibroblast growth factor, epithelial growth factor and porcine heparin. Murine lymphoma cell line EL4 was obtained from Sigma and was maintained in DMEM with FCS 
A
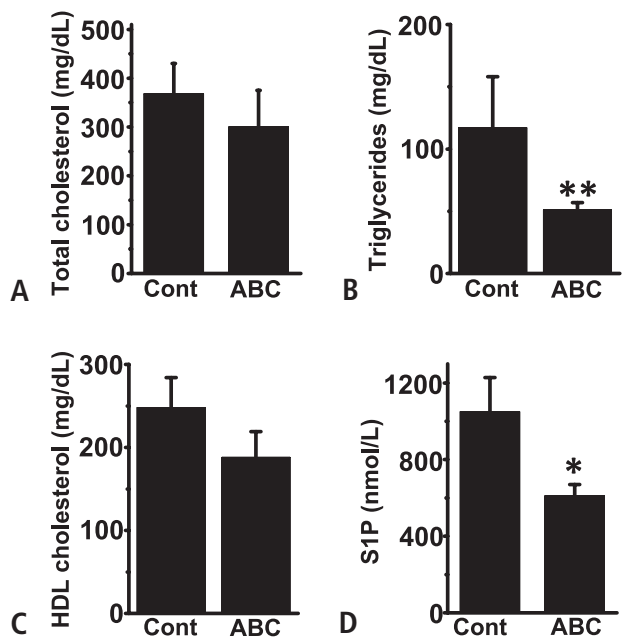

Figure 1: Effect of $A B C 294640$ on plasma lipid levels. $L D L-R^{-/-}$mice were placed on a Western-type diet and saline ( $n=12$; Cont) or $A B C 294640$

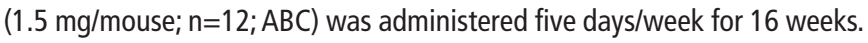
Plasma was obtained after completion of treatment, and total cholesterol (A), triglyceride (B), HDL-cholesterol (C), and S1P (D) levels were determined as described under Material and methods. ${ }^{*}-p<0.05,{ }^{*}{ }^{*}-p<0.01$ ( $A B C 294640$ vs. control).

$(10.0 \%, \mathrm{v} / \mathrm{v})$. For the assessment of lymphocyte adhesion, EL4 cells were labelled for $30 \mathrm{~min}$ at $37^{\circ} \mathrm{C}$ with fluorescent dye calcein (1.0 $\mathrm{\mu M}$, Molecular Probes) and added at a number of $4 \times 10^{6} \mathrm{cells} /$ $\mathrm{ml}$ to a confluent bEnd. 5 monolayer for 30 min under gentle rocking. Non-adherent cells were removed thereafter by rinsing plates three times and the number of adherent cells was counted under fluorescence microscope Leica DM-IRE (Leica Mikrosysteme, Wetzlar, Germany) with at least five fields per cover slip being quantified.

\section{Western blotting}

Endothelial cells were lysed in a buffer containing $50 \mathrm{mM}$ Tris$\mathrm{HCl}, 0.15 \mathrm{M} \mathrm{NaCl}, 1.0 \%$ (v/v) Nonidet P-40, 5.0\% (v/v) sodium deoxycholate, $0.1 \%(\mathrm{v} / \mathrm{v})$ SDS, $300 \mathrm{mM} \mathrm{NaF}, 1.0 \mathrm{mM}$ EGTA, $1.0 \mathrm{mM}$ orthovanadate, and protease inhibitors (Complete, Roche). Celllysates $(50 \mu \mathrm{g} / \mathrm{lane})$ were subjected to SDS-polyacrylamide gel electrophoresis. Thereafter proteins were transferred to nitrocellulose membranes, which were blocked overnight in Trisbuffered saline containing 5.0\% non-fat dry milk prior to incubations with antibodies against VCAM-1 and ICAM-1(both ABBiotec, San Diego, CA, USA).

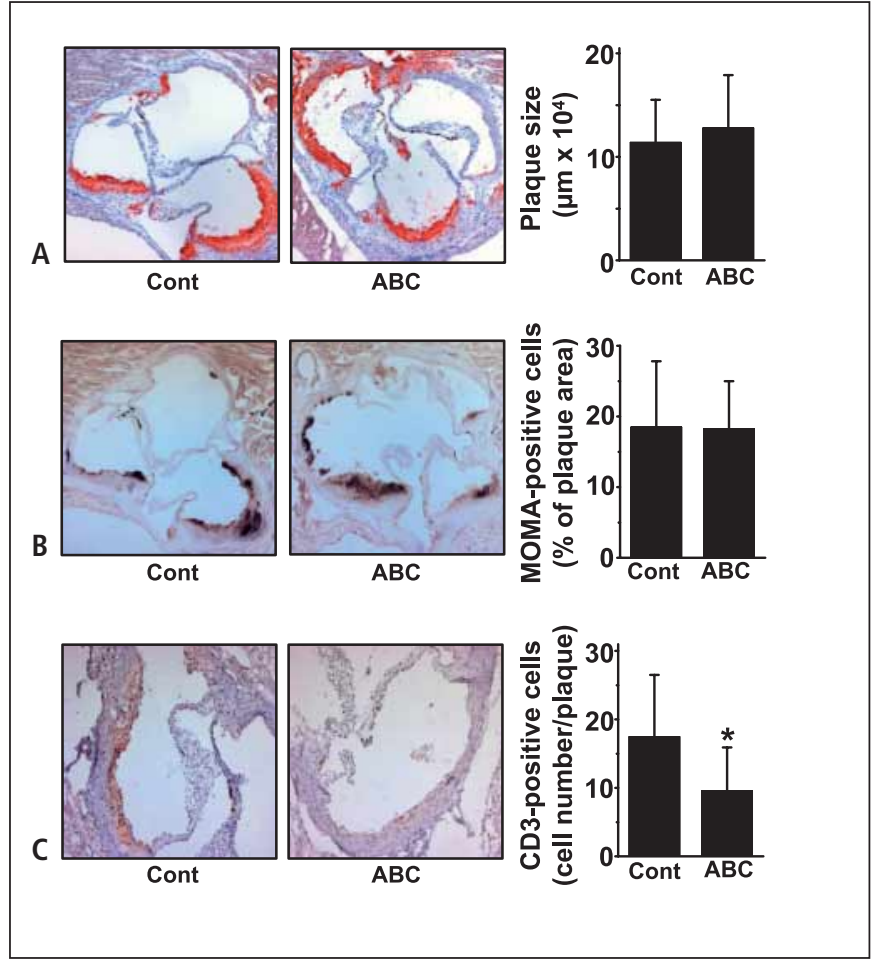

Figure 2: Effect of $A B C 294640$ on lesion development in $L D L-R^{-1-}$ mice. Western-type diet-fed $L D L-R^{-/}$mice were administered saline $(n=12$; Cont) or ABC294640 (1.5 mg/mouse; $n=12 ; A B C)$ for 16 weeks, euthanised, bled and aortic roots were fixed, sectioned and used for morphometric analysis or stained for macrophages (MOMA-2) or T-cells (CD3). A) Left panel - representative Oil Red 0 staining of aortic root lesions from control and ABC294640-treated mice. Right panel - quantification of atherosclerotic lesion area. B) Left panel - representative MOMA-2 staining $(x 200)$ from control and ABC294640 treated mice. Right panel - bar graph showing the content of macrophages in the atherosclerotic plaque that was calculated as percentage of staining area relative to the total plaque size. C) Left panel demonstration of CD3 staining in atherosclerotic plaque. Right panel - bar graph showing the number of CD3-positive cells within the plaque area. * $p<0.05$ ( $A B C 294640$ vs. control).

\section{Statistical analysis}

Data are presented as means \pm standard deviation (SD) for at least three separate experiments or as results representative for at least three repetitions. Comparisons between the means of two groups were performed with two-tailed Student t-test. Comparisons between the means of multiple groups were made by one-way ANOVA for independent samples. Pairwise comparisons were performed thereafter with Student-Newman-Keuls post-hoc test. P-values less than 0.05 were considered significant. 
A

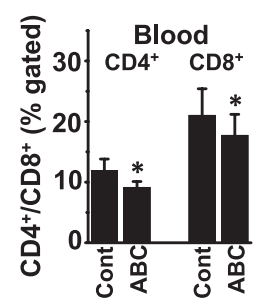

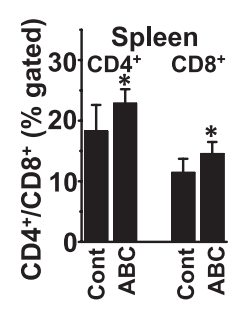

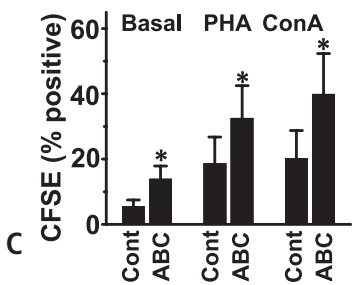

B

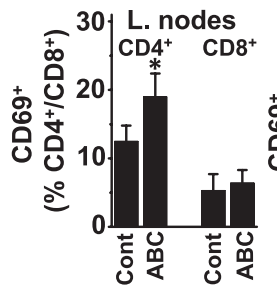

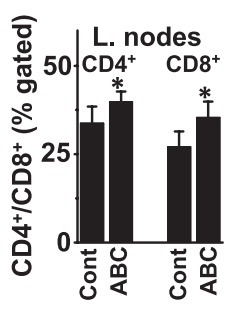

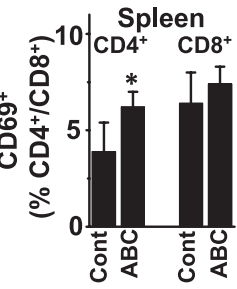

c

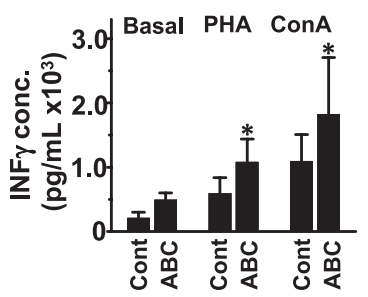

Figure 3: Effect of $A B C 294640$ on lymphocyte distribution and function in LDL- ${ }^{-1-}$ mice. Western-type diet-fed LDL- $\mathrm{R}^{-/-}$mice were administered saline ( $n=12$; Cont) or ABC294640 (1.5 mg/mouse; $n=12 ; A B C)$ for 16 weeks, euthanised, bled and splenocytes and lymph node cells were isolated as described under Material and methods. A and B) Whole blood (A) or single cell suspensions ( $A$ and $B$ ) were stained with antibodies against CD4, CD8 or CD69 and analysed by flow cytometry. Data are presented as relative cell numbers. $C$ and $D$ ) Splenocytes were seeded in 96-well plates and stimulated for $96 \mathrm{~h}$ with ConA $(10.0 \mu \mathrm{g} / \mathrm{ml})$ or PHA $(5.0 \mu \mathrm{g} / \mathrm{ml})$. Cell proliferation was determined by flow cytometry as CFSE dilution effect (C). Cytokine concentrations in supernatants were determined by ELISA (D). ${ }^{*}-p<0.05$ (ABC294640 vs. control).

\section{Results}

\section{SphK inhibition reduces plasma triglycerides and S1P levels}

$\mathrm{ABC} 294640$ is a synthetic SphK inhibitor with $\mathrm{IC}_{50}$ of $60.0 \mu \mathrm{M}$ for SphK2 and above $100 \mu \mathrm{M}$ for SphK1, and that can be chronically administered to mice without systemic toxicity (21). Here, ABC294640 was administered five-weekly via i.p. injections to eight-week-old LDL- $\mathrm{R}^{-/-}$mice for a period of 16 weeks at a dose previously demonstrated to effectively suppress dextran sulfateinduced colitis and diabetic rethinopathy $(22,26)$. Body weight did not differ between groups when fed a cholesterol-enriched Western type diet (not shown). Similarly, both total and HDL cholesterol remained unaltered ( Fig. 1). However, there was a considerable decrease in triglyceride levels in the ABC294640-treated group. As expected, administration of ABC294640 significantly reduced plasma S1P levels by approximately $30 \%$.

\section{SphK inhibition fails to affect plaque size but alters cellular composition}

Lesion size was measured to determine the effect of SphK inhibition on the diet-induced atherogenesis. Morphometric analysis of the Oil Red O-stained aortic root did not show any effect of ABC294640 on the absolute area of the plaque ( Fig. 2A) or on the plaque-to-lumen ratio (not shown). Immunohistochemical analysis of lesion composition yielded no significant differences with regard to MOMA-positive macrophage content ( Fig. 2B). Further analysis, however, showed that treatment with ABC294640 substantially reduced plaque content of CD3-positive T cells - an important determinant of the intraplaque inflammation ( Fig. 2c).

\section{SphK inhibition affects lymphocyte profile and enhances T cell activation}

Numerous studies revealed regulatory influence of S1P on lymphocyte distribution and T cell function $(8,9)$. Therefore, we next assessed the effect of SphK inhibition on lymphocyte subsets in blood and lymphatic organs. Blood lymphocyte count remained unaltered in treated animals as compared to controls (data not shown). However, ABC294640 treatment led to a slight but significant decrease of $\mathrm{CD}^{+} \mathrm{T}$ and $\mathrm{CD} 8^{+} \mathrm{T}$ cell levels in blood, while the corresponding cell counts in lymph nodes and spleen were increased ( Fig. 3A). In addition, lymph node and spleen $\mathrm{CD} 4^{+} \mathrm{T}$ cells (but not $\mathrm{CD} 8^{+} \mathrm{T}$ cells) were enriched in CD69, an early activation marker and a putative lymphatic retention signal ( Fig. 3B). SphK inhibition did not noticeably influence total B cell (CD19) numbers in blood, lymph nodes and spleen (data not shown). In addition to lymphocyte count and subset pattern, we also addressed the influence of SphK inhibition on lymphocyte 

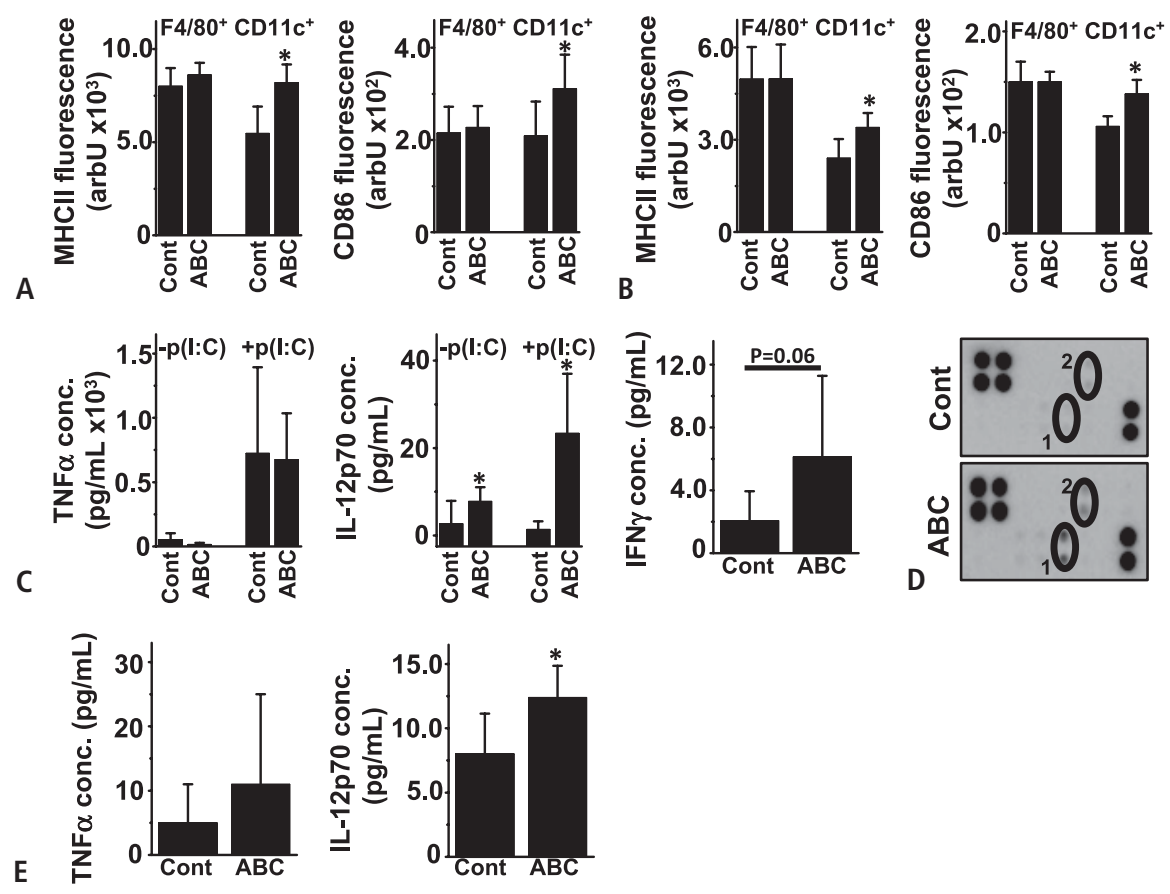

Figure 4: Effect of ABC294640 on macrophage and dendritic cell activation in LDL- $R^{-/-}$mice. Western-type diet-fed LDL- $\mathrm{R}^{-/-}$mice were administered saline ( $n=12$; Cont) or $A B C 294640$ ( $1.5 \mathrm{mg} /$ mouse; $n=12 ; A B C)$ for 16 weeks, euthanised, bled and peritoneal cells and splenocytes were isolated as described under Material and methods. A and B) Single splenocyte (A) or peritoneal cell (B) suspensions were stained with antibodies against $F 4 / 80$, CD11C, MHCII or CD86 and analysed by flow cytometry. Data are presented as fluorescence intensity. C) Peritoneal macrophages were established in cul- ture, incubated for $24 \mathrm{~h}$ in the absence or presence of poly $(\mathrm{l}: \mathrm{C})(50.0 \mathrm{ng} / \mathrm{ml})$ and TNF $\alpha$, IL12p70 and IFN $\gamma$ concentrations in the cell medium were determined using ELISA. D) Cytokine profile of pooled macrophage supernatants from six mice of control or ABC294640 treatment groups was determined semi-quantitatively by a RayBiotech cytokine array. Shown are arrays representative of one out of two determinations. 1. RANTES; 2. IL-4. E) Plasma levels of TNF $\alpha$ and IL12p70 were determined in each animal by ELISA. * $p<0.05$ ( $A B C 294640$ vs. control).

antigen-presenting cells has been examined in cells positive for $\mathrm{F} / 80^{+}$(a macrophage marker) or $\mathrm{CD}_{11 \mathrm{c}^{+}}$(a dendritic cell marker). Figure $4 \mathrm{~A}$ demonstrates increased expression of MHC-II and CD86 on CD11c ${ }^{+}$but not on $\mathrm{F} 4 / 80^{+}$cells obtained from ABC294640-treated animals. Similar though less pronounced expression pattern characterised by the elevated surface presence of co-stimulatory molecules on $\mathrm{CD} 11 \mathrm{c}^{+}$cells has been also observed in splenocytes obtained from ABC294640-treated animals ( Fig. 4B). As macrophage and dendritic cell activation is associated with an altered secretion of several inflammatory mediators, we next tested for changes in the production of inflammatory cytokines and chemokines in peritoneal leukocytes brought about by ABC294640 administration. As shown in Figure 4C, concentrations of TNF $\alpha$ - a cytokine predominantly synthesised by macrophages - were similar in peritoneal cell supernantants from ABC294640-treated and control LDL- $\mathrm{R}^{-/-}$mice both in a basal state and after stimulation with poly(I:C), a Toll-like 3 (TLR3) receptor agonist. By contrast, basal and poly(I:C)-stimulated production of IL-12p70 - a cytokine synthesised in copious amounts by both macrophages and dendritic cells - was elevated in peritoneal cells obtained from ABC294640 treated animals. Moreover, these cells were characterised by increased basal release of
As $\mathrm{T}$ cell activation is controlled by antigen presenting cells, we next examined the effect of SphK inhibition on the function of macrophages and dendritic cells. To this purpose, peritoneal leukocytes were obtained from control and ABC294640-treated animals and the surface expression of co-stimulatory molecules (MHC class II, CD86) defining the subpopulation of activated 


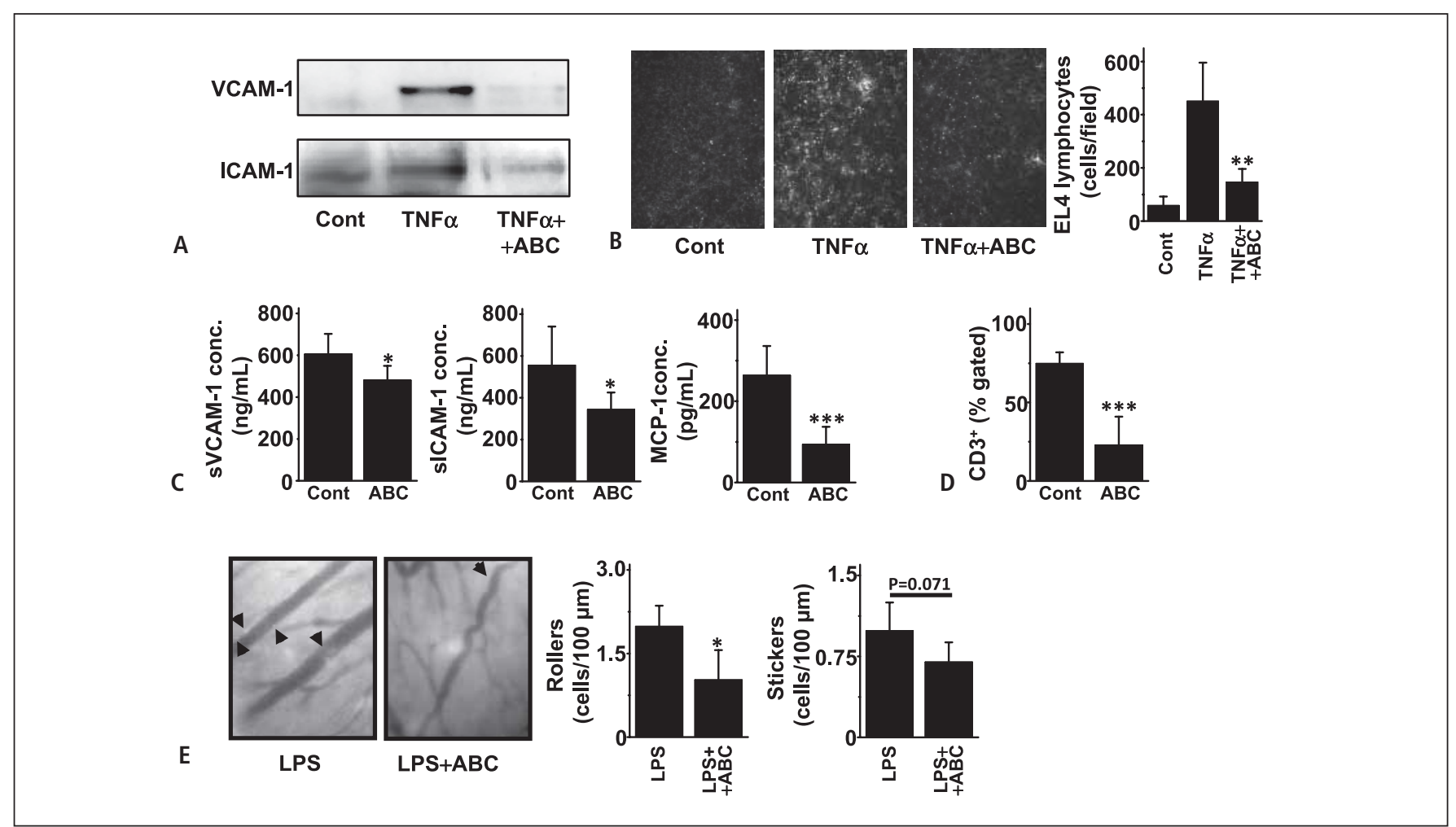

Figure 5: Effect of $A B C 294640$ on endothelial cell activation in vitro and in vivo. $A$ and $B$ ) bEnd3 murine endothelial cells were stimulated with TNF $\alpha(50.0 \mathrm{ng} / \mathrm{ml})$ for $4 \mathrm{~h} . \mathrm{VCAM}-1$ and ICAM-1 expression was determined using Western blot $(A)$ and the adherence of calcein-loaded EL4 murine lymphocyte was observed under fluorescence microscope (B). Data are representative of three independent experiments. Right panel - quantification of EL4 lymphocyte adhesion to bEnd. 3 cells. C) Western-type diet-fed LDL- $\mathrm{R}^{-1-}$ mice were administered saline ( $n=12$; Cont) or $A B C 294640$ (1.5 mg/mouse; $n=12$; ABC) for 16 weeks. Plasma levels of soluble VCAM-1 (sVCAM-1), ICAM-1 (sICAM-1), and MCP-1 were determined in each animal by ELISA. D and E)

IFN $\gamma$, which is produced by dendritic cells but not by macrophages. The extended analysis of cytokine and chemokine production using a semi-quantitative cytokine array additionally revealed that peritoneal cell supernatants from $\mathrm{ABC} 294640$ treated LDL- $\mathrm{R}^{-/}$mice were enriched in RANTES and IL-4 ( Fig. 4D). The modification of cyto- and chemokine production profile evoked in peritoneal cells by $\mathrm{ABC} 294640$ administration was paralleled by respective changes in plasma cytokine concentrations. As shown in - Figure $4 \mathrm{E}, \mathrm{ABC} 294640$ treatment of $\mathrm{LDL}-\mathrm{R}^{-/}$mice led to a marked elevation of the plasma levels of IL-12p70, while levels of $\mathrm{TNF} \alpha$ remained unaltered.

\section{SphK inhibition improves anti-adhesive properties of endothelial cells}

As lymphocyte recruitment to arteriosclerotic lesions depends on the functional integrity of endothelium, we next investigated the
Western-type diet-fed LDL- $\mathrm{R}^{-/-}$mice were administered saline $(\mathrm{n}=8$; Cont) or $A B C 294640$ (1.5 mg/mouse; $n=8 ; A B C)$ for four weeks. The relative number of $\mathrm{CD}^{+}$cells in peritoneal cell suspension was determined in three animals $12 \mathrm{~h}$ after CCL1 9 injection (D). Tethering and adherence of perivitally labelled leukocytes to capillary vessel wall was examined after a single dose of LPS $(5.0 \mathrm{mg} / \mathrm{kg})$ in five animals using intravital microscopy as described in Material and methods (E). Left panel: Original photographs of peritoneal arterioles. Arrows indicate fluorescently labelled adherent leukocytes. ${ }^{*}$ $p<0.05 ;{ }^{* *}-p<0.01 ;{ }^{* * *}-p<0.001$ (ABC294640 vs. control).

effects of SphK inhibition on the anti-adhesive properties of endothelial cells. To this aim, bEnd.5 murine endothelial cells were pre-incubated for $1 \mathrm{~h}$ with $50.0 \mu \mathrm{M} \mathrm{ABC} 294640$ and afterwards were exposed to $100 \mathrm{U} / \mathrm{ml}$ of TNF $\alpha$. As shown in Figure $5 \mathrm{~A}$, TNF $\alpha$ potently induced expression of adhesion molecules VCAM-1 and ICAM-1 in the absence but not in the presence of ABC294640. The extent of lymphocyte adhesion to the endothelium was examined next using an in vitro cytoadherence assay. - Figure 5B demonstrates the increased adhesion of EL4 murine lymphocytes to bEnd. 5 endothelial cells exposed to $100 \mathrm{U} / \mathrm{ml}$ of TNF $\alpha$ and its almost complete reversal by pre-treatment of cells with ABC294640 $(50.0 \mu \mathrm{M})$. To investigate the anti-adhesive properties of ABC294640 under in vivo conditions, we initially determined plasma concentrations of soluble adhesion molecules. As shown in Figure 5C, administration of ABC294640 to LDL- $\mathrm{R}^{-/-}$ mice significantly reduced both sVCAM- 1 and sICAM- 1 levels in plasma. In addition, the plasma concentration of MCP-1 - a chemoattractant chemokine secreted by endothelial cells - was reduced in ABC294640-treated animals. We next sought to investi- 
gate, whether SphK inhibition affects the T cell capacity to egress the bloodstream and penetrate through endothelial barrier to extravascular space. As shown in Figure 5D, i.p. injection of CCL19 induced the mobilisation of $\mathrm{CD}^{+} \mathrm{T}$ cells to peritoneum in control $\mathrm{LDL}^{-\mathrm{R}^{-/}}$mice, while this effect was substantially reduced in ABC294640-treated animals. Finally, leukocyte-endothelial interaction has been directly assessed in vivo in postcapillary venoles. To this aim, leukocytes were perivitally labelled with fluorescence dye (CFDA) in control and ABC294640-treated LDL- $\mathrm{R}^{-/-}$mice, and cell rolling on and tethering to the vascular wall was monitored using intravital microscopy. Figure 5E demonstrates that ABC294640 administration substantially reduced leukocyte-endothelial interaction: the number of rollers was significantly reduced while the number of stickers tended to decrease in ABC294640 treated LDL- $\mathrm{R}^{-/-}$mice as compared to controls.

\section{Discussion}

S1P signalling is instrumental in the pathogenesis of several inflammatory diseases including ulcerating colitis, viral myocarditis, endotoxin-induced lung injury, or autoimmune encephalomyelitis (27-30). Recent studies showed that FTY720 - a synthetic S1P mimetic interacting with $\mathrm{S} 1 \mathrm{P}$ receptor types present in vasculature - exerts anti-atherogenic effects in mouse models of disease (19, 20). However, the relevance of the endogenous S1P for the development of atherosclerosis has not been investigated to date. Therefore, it was the aim of the present study to assess the formation of the atherosclerotic lesions under conditions of SphK inhibition and reduced S1P formation. To suppress endogenous S1P synthesis we used ABC294640, a synthetic SK inhibitor that targets SphK2 at lower and SphK1 at higher concentrations, accumulates and reduces SphK activity in peripheral tissues, and shows anti-inflammatory and anti-tumor activities in murine models of diseases $(21,22,26)$. In our hands, ABC294640 decreased plasma S1P levels by approximately $30-40 \%$. This is comparable to the effect of SphK2 gene knock-out in mouse, in which both reduced plasma S1P levels and SphK activity were observed (31). However, neither absolute nor fractional lesion area was affected in LDL- $\mathrm{R}^{-/-}$mice treated with ABC294640.

The lowering effect of SphK inhibition on triglycerides is for the first time reported here and is rather unexpected in light of previous investigations, which failed to register any plasma lipid and lipoprotein alterations in mice treated with synthetic S1P mimetics $(19,20)$. However, increased generation of triglyceride-rich lipoproteins has been observed in mice with haematopoietic deficiency of S1P degrading enzyme, S1P lyase, which are characterised by substantially increased endogenous S1P levels in plasma (Bot M, unpublished observation). In addition, S1P was found to affect lipolysis in differentiated rat white adipocytes (32). Collectively, these observations point to the modulatory effect of S1P on triglyceride production and/or degradation, and mandate further research to clarify its role in the regulation of intermediary lipid metabolism.
In contrast to lipid metabolism, the profound regulatory impact of S1P on T cell distribution and function is well established $(8,9)$. Formation of increasing S1P concentration gradients between the lymphoid tissue and the adjacent blood is essential for the effective lymphocyte egress and recirculation, and the retention of T cells in spleen and lymph nodes was observed after S1PR1 elimination with genetic or pharmacologic approaches (33-35). Moreover, S1P negatively regulates cell surface expression of CD69 - an early activation marker and a putative retention signal within lymphoid organs (36). Functional studies revealed reduced proliferation and cytokine secretion in vitro by $\mathrm{T}$ cells exposed to S1P or in vivo after adoptive transfer of lymphocytes constitutively overexpressing S1PR (37-39). Consistent with these observations, the present study documents slight but significant redistribution of $\mathrm{CD}^{+} \mathrm{T}$ cells and $\mathrm{CD} 8^{+} \mathrm{T}$ cells from blood to spleen and lymph nodes that might be expected after reduction of the plasma S1P concentration and the plasma-lymphoid tissue S1P gradient. Moreover, the protracted inhibition of SphK activity led to the substantial enhancement of $\mathrm{T}$ cell activity as evidenced by the increased CD69 expression on the cell surface (predominantely on $\mathrm{CD} 4^{+} \mathrm{T}$ cells) as well as the proliferation and the cytokine production in response to ConA and PHA stimulation. The latter effects might arise in consequence of toning down the inhibitory influence exerted directly by S1P on T cell functions or by the increased activity of antigen presenting cells, which are vital for boosting T cell activity. Actually, the present study for the first time provides evidence for the increased surface expression of costimulatory molecules (CD86, MHC class II) reflecting the immunostimulatory activity on $\mathrm{CD} 11 \mathrm{c}^{+}$dendritic cells under conditions of chronic SphK inhibition. In addition, continuous treatment with SphK inhibitor was accompanied by increased plasma levels and/or production by peritoneal cells of IL-12p70 and IFN $\gamma$ - cytokines synthesised in copious amounts by dendritic cells. Our in vivo findings corroborate results of several previous in vitro studies, which demonstrated inhibitory effects of both S1P and synthetic S1P mimetics on chemotactic responses and cytokine production by dendritic cells as well as on their capacity to serve as antigen-presenting cells (40-42). In particular, the increased production of IL-12p70 in SphK1 deficiency and its reversal by S1P agonists have been recently reported in dendritic cells differentiated from splenocytes (43). Taken together, the present data point to the persistent hyperactivation of dendritic cell-T cell axis under condition of chronic SphK inhibition and reduced endogenous S1P formation.

$\mathrm{T}$ cells are highly involved in the development of atherosclerosis. Both $\mathrm{CD} 4^{+} \mathrm{T}$ cells and $\mathrm{T}$ cell-derived cytokines are present throughout human and murine plaques and the adaptive immunity deficiency or the selective $\mathrm{T}$ cell deficiency both lead to reduced atherosclerosis in mice $(44,45)$. Similarly, dendritic cells are found in atherosclerotic lesions, where they are believed to orchestrate $\mathrm{CD} 4^{+} \mathrm{T}$ cells infiltration, priming, and clonal expansion (46). Given the crucial role of $\mathrm{T}$ cells and dendritic cells in atherosclerosis, the unaltered lesion burden in SphK inhibitor-treated animals is surprising and suggests that some anti-atherogenic mechanisms instigated by SphK inhibition might countervail pro- 
atherogenic effects related to dendritic cell-T cell axis hyperactivation. Previous studies revealed that SphK plays a critical role in mediating endothelial activation and induction of adhesion molecules in response to stimulation with pro-inflammatory factors such as TNF $\alpha$ or high glucose concentrations $(11,47)$. Actually, suppression of SphK activity with an unspecific inhibitor N'N'-dimethylsphingosine (DMS) was shown to reduce both the expression of ICAM-1, VCAM-1, and E-selectin and the adherence of monocytes to human endothelial cells, while overexpression of SphK1 leading to augmented intracellular S1P generation exerted opposite effects $(11,48)$. In the present study, we recapitulate these findings in the murine endothelium using a specific SphK inhibitor and extend them by showing the decreased endothelial adhesion of a lymphocyte cell line. In addition, we for the first time demonstrate that the inhibition of SphK reduce the tethering and the adhesion of leukocytes to the vascular wall under in vivo conditions and that the latter effect translates into diminished mobilisation of T cells from blood to extravascular compartment (peritoneal cavity) and possibly to atherosclerotic lesion, where lower $\mathrm{T}$ cell numbers have been observed (see Fig. 2C). Taking into account that the induction of surface adhesion molecules represents a key cellular phenomenon initiating and facilitating vascular inflammation leading to the development of atherosclerosis, the reduced leukocyte adherence in ABC296640-treated mice provides a plausible explanation for the absence of accelerated lesion formation in these animals in spite of decreased extracellular S1P levels.

Both pro- and antiatherogenic effects have been attributed to $\mathrm{S} 1 \mathrm{P}$ and the exact role fulfilled by this compound in the pathogen-

\section{What is known about this topic?}

- Sphingosine 1-phosphate (S1P) is a bioactive sphingolipid and a constituent of high density lipoproteins that account for several anti-atherogenic effects of these lipoproteins in vitro.

- Synthetic mimetics of S1P such as FTY720 reduce atherosclerosis in animal models of disease (apoE-deficient mice, LDL-R-deficient mice).

- Anti-atherogenic effects of endogenously produced S1P have not been as yet investigated in vivo.

\section{What does this paper add?}

- We here for the first time examined development of atherosclerosis under conditions of sphingosine kinase inhibition and reduced generation of endogenous S1P.

- We demonstrate that inhibition of sphingosine kinase leads to both pro-atherogenic effects (via enhanced activation of dendritic cells and T-cells) and anti-atherogenic effects (via reduced activation of endothelial cells) in LDL-R-deficient mice. As a consequence, sphingosine kinase inhibition does not affect atherosclerotic lesion formation.

- This study highlights the complex interplay between S1P metabolism and signalling and diverse processes involved in the development of atherosclerosis. esis of atherosclerosis remains a matter of lively debate. Most of the putative anti-atherogenic effects of S1P including stimulation of endothelial proliferation, migration, survival, and NO production as well as inhibition of pro-inflammatory activities of macrophages and lymphocytes are attributed to the ligation of $G$ proteincoupled S1P receptors $(4,5)$. However, S1P acts also as a second messenger, which is formed by SphK downstream of extracellular signals such as pro-inflammatory cytokines and mediates direct intracellular effects via Ras, mitogen-activating protein kinase and/or $\mathrm{Ca}^{2+}$ currents $(5,10)$. The outcomes of intracellular S1P signalling, which encompass activation of the transcription factor nuclear factor- $\kappa \mathrm{B}$ as well as expression of cytokines such as TNF $\alpha$ and adhesion-mediating molecules such as VCAM-1 and ICAM-1 in macrophages and endothelial cells, respectively, may be considered pro-atherogenic. Based on these findings the hypothesis has been previously put forward that extra- and intracellular S1P signalling play opposite roles in the atherosclerosis development $(49,50)$. The results of the present study, which demonstrate the simultaneous occurrence of pro- and anti-atherogenic effects under conditions of SphK inhibition and reduced S1P generation, lend for the first time experimental support to this hypothesis.

The present study has certain limitations. First, using ABC296640 as SphK inhibitor led to reduction of plasma S1P by approx. 30\%, which may not be sufficient to fully expose its positive or negative influence on the progression of atherosclerosis. Second, as ABC294640 targets SphK1 and SphK2 at different concentrations, it cannot be excluded that both isoforms were not equipotently inhibited in course of the treatment. These limitations may additionally explain the neutral effects of ABC296640 on the development of atherosclerosis as opposed to clearly antiatherogenic effects exerted by potent synthetic agonists of S1P receptors $(19,20)$. The present findings mandate further investigations of the effect of endogenous S1P on the development of atherosclerosis exploiting more potent and specific inhibitors or knock-outs of enzymes involved in S1P synthesis or degradation. In this context, it may be worth noticing that hematopoietic S1P lyase deficiency elevates endogenous S1P levels in plasma and decreases atherosclerotic lesion formation in $\mathrm{LDL}^{-\mathrm{R}^{-/}}$mice (Bot $\mathrm{M}$, unpublished observation).

In conclusion, treatment with SphK inhibitor leads to both proand anti-atherogenic effects in LDL- $\mathrm{R}^{-/}$mice. As a consequence, SphK inhibition fails to affect atherosclerosis despite significant $\mathrm{S} 1 \mathrm{P}$ reduction in plasma.

\section{Acknowledgements}

The authors would like to thank Dr. Ilse Bot for the most helpful discussion. The expert technical assistance of Beate Schulte, Cornelia Richter-Elsenheimer, Anna Leroy, and Katrin Tkotz is gratefully acknowledged.

\section{Conflict of interests}

Dr. Lynn Maines is Vice-President of Apogee Biotechnology Corp. 


\section{References}

1. Strub GM, Maceyka M, Hait NC, et al. Extracellular and intracellular actions of sphingosine-1-phosphate. Adv Exp Med Biol 2010; 688: 141-155.

2. Zhang B, Tomura H, Kuwabara A, et al. Correlation of high density lipoprotein (HDL)-associated sphingosine 1-phosphate with serum levels of HDL-cholesterol and apolipoproteins. Atherosclerosis 2005; 178: 199-205.

3. Alemany R, van Koppen CJ, Danneberg K, et al. Regulation and functional roles of sphingosine kinases. Naunyn Schmiedebergs Arch Pharmacol 2007; 374: 413-428.

4. Takabe K, Paugh SW, Milstien S, et al. „Inside-out“ signaling of sphingosine1-phosphate: therapeutic targets. Pharmacol Rev 2008; 60: 181-195.

5. Rosen H, Gonzalez-Cabrera PJ, Sanna MG, et al. Sphingosine 1-phosphate receptor signaling. Annu Rev Biochem 2009; 78: 743-768.

6. Lucke S, Levkau B. Endothelial functions of sphingosine-1-phosphate. Cell Physiol Biochem 2010; 26: 87-96.

7. Igarashi J, Michel T. Sphingosine-1-phosphate and modulation of vascular tone. Cardiovasc Res 2009; 82: 212-220.

8. Cyster JG. Chemokines, sphingosine-1-phosphate, and cell migration in secondary lymphoid organs. Annu Rev Immunol 2005; 23: 127-159.

9. Rivera J, Proia RL, Olivera A. The alliance of sphingosine-1-phosphate and its receptors in immunity. Nat Rev Immunol 2008; 8: 753-763.

10. Weigert A, Weis N, Brüne B. Regulation of macrophage function by sphingosine1-phosphate. Immunobiology 2009; 214: 748-760.

11. Xia P, Gamble JR, Rye KA, et al. Tumor necrosis factor-alpha induces adhesion molecule expression through the sphingosine kinase pathway. Proc Natl Acad Sci USA 1998; 95: 14196-14201.

12. Zhi L, Leung BP, Melendez AJ. Sphingosine kinase 1 regulates pro-inflammatory responses triggered by TNFalpha in primary human monocytes. J Cell Physiol 2006; 208: 109-115.

13. Sattler KJ, Elbasan S, Keul P, et al. Sphingosine 1-phosphate levels in plasma and HDL are altered in coronary artery disease. Basic Res Cardiol 2010; 105: 821-832.

14. Argraves KM, Sethi AA, Gazzolo PJ, et al. S1P, dihydro-S1P and C24: 1-ceramide levels in the HDL-containing fraction of serum inversely correlate with occurrence of ischemic heart disease. Lipids Health Dis 2011; 10: 70.

15. Argraves KM, Argraves WS. HDL serves as a S1P signaling platform mediating a multitude of cardiovascular effects. J Lipid Res 2007; 48: 2325-2333.

16. Nofer JR. High-density lipoprotein, sphingosine 1-phosphate, and atherosclerosis. J Clin Lipidol 2008; 2: 4-11.

17. Rodríguez C, González-Díez M, Badimon L, et al. Sphingosine-1-phosphate: A bioactive lipid that confers high-density lipoprotein with vasculoprotection mediated by nitric oxide and prostacyclin. Thromb Haemost 2009; 101: 665-673.

18. González-Díez M, Rodríguez C, Badimon L, et al. Prostacyclin induction by highdensity lipoprotein (HDL) in vascular smooth muscle cells depends on sphingosine 1-phosphate receptors: effect of simvastatin. Thromb Haemost 2008; 100: 119-126.

19. Nofer JR, Bot M, Brodde M, et al. FTY720, a synthetic sphingosine 1 phosphate analogue, inhibits development of atherosclerosis in low-density lipoprotein receptor-deficient mice. Circulation 2007; 115: 501-508.

20. Keul P, Tölle M, Lucke S, et al. The sphingosine-1-phosphate analogue FTY720 reduces atherosclerosis in apolipoprotein E-deficient mice. Arterioscler Thromb Vasc Biol 2007; 27: 607-613.

21. French KJ, Zhuang Y, Maines LW, et al. Pharmacology and antitumor activity of ABC294640, a selective inhibitor of sphingosine kinase-2. J Pharmacol Exp Ther 2010; 333: 129-139.

22. Maines LW, Fitzpatrick LR, French KJ, et al. Suppression of ulcerative colitis in mice by orally available inhibitors of sphingosine kinase. Dig Dis Sci 2008; 53 : 997-1012.

23. Yusof M, Kamada K, Gaskin FS, et al. Angiotensin II mediates postischemic leukocyte-endothelial interactions: role of calcitonin gene-related peptide. Am J Physiol Heart Circ Physiol 2007; 292: H3032-H3037.

24. Nofer JR, van der Giet M, Tölle M, et al. HDL induces NO-dependent vasorelaxation via the lysophospholipid receptor S1P3. J Clin Invest 2004; 113: 569-581.

25. Varga G, Balkow S, Wild MK, et al. Active MAC-1 (CD11b/CD18) on DCs inhibits full T-cell activation. Blood 2007; 109: 661-669.

26. Maines LW, French KJ, Wolpert EB, et al. Pharmacologic manipulation of sphingosine kinase in retinal endothelial cells: implications for angiogenic ocular diseases. Invest Ophthalmol Vis Sci 2006; 47: 5022-5031.
27. Miyamoto T, Matsumori A, Hwang MW, et al. Therapeutic effects of FTY720, a new immunosuppressive agent, in a murine model of acute viral myocarditis. J Am Coll Cardiol 2001; 37: 1713-1718.

28. Peng X, Hassoun PM, Sammani S, et al. Protective effects of sphingosine 1-phosphate in murine endotoxin-induced inflammatory lung injury. Am J Respir Crit Care Med 2004; 169: 1245-1251.

29. Webb M, Tham CS, Lin FF, et al. Sphingosine 1-phosphate receptor agonists attenuate relapsing-remitting experimental autoimmune encephalitis in SJL mice. J Neuroimmunol 2004; 153: 108-121.

30. Fujii R, Kanai T, Nemoto Y, et al. FTY720 suppresses CD4+CD44highCD62L- effector memory T cell-mediated colitis. Am J Physiol Gastrointest Liver Physiol 2006; 291: G267-G274.

31. Kharel Y, Lee S, Snyder AH, et al. Sphingosine kinase 2 is required for modulation of lymphocyte traffic by FTY720. J Biol Chem 2005; 280: 36865-36872.

32. Jun DJ, Lee JH, Choi BH, et al. Sphingosine-1-phosphate modulates both lipolysis and leptin production in differentiated rat white adipocytes. Endocrinology 2006; 147: 5835-5844.

33. Chi H, Flavell RA. Cutting edge: regulation of T cell trafficking and primary immune responses by sphingosine 1-phosphate receptor 1. J Immunol 2005; 174: 2485-2488.

34. Matloubian M, Lo CG, Cinamon G, et al. Lymphocyte egress from thymus and peripheral lymphoid organs is dependent on S1P receptor 1. Nature 2004; 427: 355-360.

35. Allende ML, Dreier JL, Mandala S, et al. Expression of the sphingosine 1-phosphate receptor, S1P1, on T-cells controls thymic emigration. J Biol Chem 2004; 279: 15396-15401.

36. Alfonso C, McHeyzer-Williams MG, Rosen H. CD69 down-modulation and inhibition of thymic egress by short- and long-term selective chemical agonism of sphingosine 1-phosphate receptors. Eur J Immunol 2006; 36: 149-159.

37. Jin Y, Knudsen E, Wang L, et al. Sphingosine 1-phosphate is a novel inhibitor of T-cell proliferation. Blood 2003; 101: 4909-4915.

38. Dorsam G, Graeler MH, Seroogy C, et al. Transduction of multiple effects of sphingosine 1-phosphate (S1P) on T cell functions by the S1P1 G proteincoupled receptor. J Immunol 2003; 171: 3500-3507.

39. Gräler MH, Huang MC, Watson S, et al. Immunological effects of transgenic constitutive expression of the type 1 sphingosine 1-phosphate receptor by mouse lymphocytes. J Immunol 2005; 174: 1997-2003.

40. Müller H, Hofer S, Kaneider N, et al. The immunomodulator FTY720 interferes with effector functions of human monocyte-derived dendritic cells. Eur J Immunol 2005; 35: 533-545.

41. Gollmann G, Neuwirt H, Tripp CH, et al. Sphingosine-1-phosphate receptor type-1 agonism impairs blood dendritic cell chemotaxis and skin dendritic cell migration to lymph nodes under inflammatory conditions. Int Immunol 2008; 20: 911-923.

42. Lan YY, Tokita D, Wang Z, et al. Sphingosine 1-phosphate receptor agonism impairs skin dendritic cell migration and homing to secondary lymphoid tissue: association with prolonged allograft survival. Transpl Immunol 2008; 20: 88-94.

43. Schröder M, Richter C, Juan MH, et al. The sphingosine kinase 1 and S1P1 axis specifically counteracts LPS-induced IL-12p70 production in immune cells of the spleen. Mol Immunol 2011; 48: 1139-1148.

44. Gotsman I, Sharpe AH, Lichtman AH. T-cell costimulation and coinhibition in atherosclerosis. Circ Res 2008; 103: 1220-1231.

45. Ait-Oufella H, Taleb S, Mallat Z, et al. Cytokine network and T cell immunity in atherosclerosis. Semin Immunopathol 2009; 31: 23-33.

46. Niessner A, Weyand CM. Dendritic cells in atherosclerotic disease. Clin Immunol 2010; 134: 25-32.

47. Wang L, Xing XP, Holmes A, et al. Activation of the sphingosine kinase-signaling pathway by high glucose mediates the proinflammatory phenotype of endothelial cells. Circ Res 2005; 97: 891-899.

48. Limaye V, Xia P, Hahn C, et al. Chronic increases in sphingosine kinase-1 activity induce a pro-inflammatory, pro-angiogenic phenotype in endothelial cells. Cell Mol Biol Lett 2009; 14: 424-441.

49. Tamama K, Okajima F. Sphingosine 1-phosphate signaling in atherosclerosis and vascular biology. Curr Opin Lipidol 2002; 13: 489-495.

50. Okajima F, Sato K, Kimura T. Anti-atherogenic actions of high-density lipoprotein through sphingosine 1-phosphate receptors and scavenger receptor class B type I. Endocr J 2009; 56: 317-334. 